\title{
Validation Process of a High-Resolution Database in a Pediatric Intensive Care Unit - Describing the Perpetual Patient's Validation
}

\author{
Audrey Mathieu ${ }^{1}$, Michael Sauthier ${ }^{2}$, Philippe Jouvet $^{3}$, Guillaume Emeriaud ${ }^{3}$, and David \\ Brossier $^{4}$ \\ ${ }^{1}$ Centre de recherche du CHU Sainte-Justine \\ ${ }^{2} \mathrm{CHU}$ Sainte-Justine \\ ${ }^{3}$ Centre Hospitalier de L'Universite de Montreal \\ ${ }^{4} \mathrm{CHRU}$ de Caen
}

May 5, 2020

\begin{abstract}
Objective: High data quality is essential to ensure the validity of clinical and research inferences based on it. However, these data quality assessments are often missing even though these data are used in daily practice and research. Our objective was to evaluate the data quality of our high-resolution electronic database (HRDB) implemented in our pediatric intensive care unit (PICU). Design: A prospective validation study of a HRDB. Setting: A 32-bed pediatric medical, surgical and cardiac PICU in a tertiary care freestanding maternal-child health center in Canada. Population: All patients admitted to the PICU with at least one vital sign monitored using a cardiorespiratory monitor connected to the central monitoring station. Interventions: None Measurements and Main Results: Between June 2017 and August 2018, data from 295 patient days were recorded from medical devices and 4,645 data points were video recorded and compared to the corresponding data collected in the HRDB. Statistical analysis showed an excellent overall correlation $(\mathrm{R} 2=1)$, accuracy $(100 \%)$, agreement (bias=0, limits of agreement $=0)$, completeness $(2 \%$ missing data) and reliability $(\mathrm{ICC}=1)$ between recorded and collected data within clinically significant pre-defined limits of agreement. Divergent points could all be explained. Conclusions: This prospective validation of a representative sample showed an excellent overall data quality.
\end{abstract}

\section{Abstract:}

Objective: High data quality is essential to ensure the validity of clinical and research inferences based on it. However, these data quality assessments are often missing even though these data are used in daily practice and research. Our objective was to evaluate the data quality of our high-resolution electronic database (HRDB) implemented in our pediatric intensive care unit (PICU).

Design: A prospective validation study of a HRDB.

Setting: A 32-bed pediatric medical, surgical and cardiac PICU in a tertiary care freestanding maternalchild health center in Canada.

Population: All patients admitted to the PICU with at least one vital sign monitored using a cardiorespiratory monitor connected to the central monitoring station.

Interventions: None

Measurements and Main Results: Between June 2017 and August 2018, data from 295 patient days were recorded from medical devices and 4,645 data points were video recorded and compared to the corresponding data collected in the HRDB. Statistical analysis showed an excellent overall correlation $\left(\mathrm{R}^{2}=1\right)$, accuracy 
$(100 \%)$, agreement (bias $=0$, limits of agreement $=0)$, completeness $(2 \%$ missing data $)$ and reliability $(\mathrm{ICC}=1)$ between recorded and collected data within clinically significant pre-defined limits of agreement. Divergent points could all be explained.

Conclusions: This prospective validation of a representative sample showed an excellent overall data quality.

Key words: Pediatrics; Critical care; Database; Electronic Health Record; Big data

\section{INTRODUCTION}

Over the past two decades, technological and computer advances were used extensively to modernize medicine and assist medical teams in daily practice, as shown by the widespread use of electronic medical records (EMR) or connected biomedical devices. While the dedicated purpose in health care services is patient management, these systems have been perceived by many scientists as a way of improving clinical research efficiency and data analysis (1-4). As a result, many medical databases (DB) have been built since the beginning of the twenty-first century (4-6). To optimize our research quality in our different fields of expertise such as respiratory physiology and the development of clinical decision support systems (CDSS) (7), we implemented in 2015 an automated electronic data gathering process in our pediatric intensive care unit (PICU) (8). This DB was designed to develop and validate virtual or synthetic patients for cardiorespiratory physiology as well as for CDSS and data-driven learning systems (8). However, a validation step of the collected data is necessary before considering this DB suitable for research purposes (9-11). Indeed, the value of research findings depends on data quality $(12,13)$. Several guidelines or frameworks were elaborated to evaluate and report the quality of DBs and national registries and to guide designers of DBs at each step of the data collection $(12,14,15)$. These documents highlighted the need to evaluate data quality, to compare dataset quality performance between them and raised the question of data validity that every scientist or clinician, as data users, deal with whether in day-to-day clinical care decision-making or in medical research $(16,17)$. However, none of these guidelines provide a detailed validation process that is entirely suitable for high resolution electronic DB (HRDB), defined as a database that collects more than one data point per minute per variable and per patient. Besides, to our knowledge, none of the HRDB published a detailed validation procedure and evaluation of the quality of the data (18-20). This article constitutes the final part of the validation process of our HRDB $(8,11)$. The purpose of this study was to assess the quality of the data include in our HRDB and to provide a generalizable validation method for all HRDB.

\section{METHODS}

This study was a prospective data quality assessment conducted in the PICU of Sainte-Justine hospital (Montreal, Canada), a pediatric 32-bed medical, surgical and cardiac ICU in a free-standing tertiary maternal-child health center. The study was performed between June 2017 and August 2018.

\section{Population}

Eligible patients were those admitted to the PICU with at least one vital sign monitored using a cardiorespiratory monitor connected to the central monitoring station. Patients were excluded if the presence of one study observer in the patient room was considered incompatible or inappropriate by the physician or the nurse in charge.

\section{Standard management}

As previously reported (8), as a standard of practice in our PICU, all physiological, therapeutic and clinical data from medical devices available at the bedside of all children admitted in the PICU were continuously collected in an organized HRDB linked to the EMR from admission to discharge of the PICU (8). Biomedical signals from the monitors were sampled and recorded every 5 seconds while data from ventilators and infusion pumps were recorded every 30 seconds. The full details of the HRDB structure were previously reported (8).

\section{Study protocol}

The study was divided in three periods of 14,16 and 17 days respectively (convenient samples): the first 
was dedicated to data from the monitors, the second to the data from the ventilators and the third to the infusion pumps. During the first period, data were collected on devices that displayed the monitored data outside of the patient's room, whereas both second and third period took place at the bedside. On every study day, a sample of $20 \%$ of the children hospitalized in the PICU that meet the inclusion criteria was randomly selected. One patient could have been included more than once. A videotape of the data displayed on the medical devices (monitors, ventilators and infusion pumps) and available at the bedside, such as heart rate or positive inspiratory pressure (Figure 1) was recorded. Each day, a time synchronization process with the automatically calibrated clocks of the hospital and the video recorder was made. Each monitor (IntelliVue MP60, MP70 and MX800, Koninklijke Philips Electronics, Amsterdam, the Netherlands) was video recorded for 30 seconds, each ventilator (Servo- ${ }^{\circledR}$, Maquet, Getinge, Sweden) for 90 seconds and each infusion pump (Infusomat ${ }^{\circledR}$, B. Braun Medical Inc, Bethlehem, Pennsylvania, U.S.) was simply photographed. Since ventilator data are recorded every 30 seconds in the HRDB, 90 seconds was enough to get at least two consecutive records in the HRDB. Because the infusion pumps parameters are only set, and not measured, static pictures were considered enough. The data displayed on the devices were then manually extracted into a spreadsheet from the pictures or at every second from the videotape. Data were periodically screened for aberrant values. These data, collected by one independent observer (AM) who was not implicated in patients' care, were considered as the reference data. Three types of data from medical devices were collected (Figure 1): 1) Physiologic signals from patient monitors (heart rate, oxygen saturation and systolic, diastolic and mean blood pressure) 2) Respiratory and ventilator parameters from the ventilator (positive end-expiratory pressure, peak inspiratory pressure, respiratory rate, respiratory minute volume) 3) Pharmacotherapy from the infusion pumps (ex: drug names and infusion rate). The corresponding HRDB data were extracted using structured query language (SQL) and used for comparison (Figure 1).

\section{Endpoints}

The primary endpoints were the absolute value of the selected variables (heart rate (HR) and pulse oximetry $\left(\mathrm{SpO}_{2}\right)$ ) recorded from the monitors. The secondary endpoints were:

- The absolute value of the selected variables recorded from the monitors when available: invasive arterial blood pressure (systolic (SBP), diastolic (DBP) and mean blood pressure (MBP)) and central venous pressure (CVP)

- The absolute value of the selected variables recorded from the ventilators: positive end-expiratory pressure (PEEP), positive inspiratory pressure (PIP), respiratory rate (RR), minute ventilation (VM), expiratory tidal volume (VE)

- The infusion rate

- The infused drugs' name

- The recording time of the data

- The missing data or the completeness of the dataset.

- Statistical analysis and features' definition

Reference data were compared to the experimental data simultaneously collected in the PICU HRDB at a specific time point for each patient. Variables were expressed as mean \pm standard deviation or median [minimal - maximal value] for continuous variables, depending on whether they followed a normal distribution (Shapiro-Wilk normality test) and count (percentage) for categorical variables. Comparisons between experimental and reference data were made by dependent tests as appropriate.

Under the concept of quality lies several features that tends to delineate the degree to which the HRDB is a true representation of the reality of the PICU's data $(14,21)$

- The accuracy is defined as the closeness of agreement between the experimental and the reference data. Accuracy refers to both trueness and precision. Trueness is expressed in terms of bias and corresponds to the difference between experimental and reference value. Precision relates to the distribution of the experimental values. The agreement between experimental and reference data were evaluated for each parameter measuring the absolute agreement, the mean difference (22) and using the Bland \& Altman 
analysis. Bias and limits of agreement were calculated with the R statistical package "BlandAltman" (23) based on both the original method (the difference of the two paired measurements was plotted against the mean of the two) and the modified one (the difference of the two paired measurements was plotted against the value of the reference data) of the Bland Altman analysis $(24,25)$. In theory, the data should not be modified between the measure (monitor) and the storage (database) and the accuracy should be perfect. However, rounding process could slightly impact accuracy evaluation. Moreover, accuracy implies more than just the data itself: metadata, such as timestamps and patient identifiers, could also impact accuracy in case of asynchrony for example. Acceptable limits of agreement were $a$ priori defined as $\pm 5 \%$ of the mean of the reference.

- The correlation, defined as the association between reference and experimental data, was evaluated by the determination coefficient $\left(\mathrm{R}^{2}\right)$.

- The reliability, defined as the degree to which measurements can be reproduced, echoes both agreement and correlation between experimental and reference data. It was evaluated by intraclass correlation coefficients (ICC) for each parameter. ICCs estimates, 95\% confidence intervals and F test results were calculated with the R statistical packages "irr"(26) and "psych" (22) using a single measurement, agreement, two-way mixed effect model (27).

- The completeness is related to the amount and the nature of the missing data and is defined as the extent to which the data that should have been included were indeed included. To evaluate the completeness, the data of infusion pumps within the HRDB were compared to the corresponding data in the EMR. We compared for each selected patient, throughout the day, the data recorded in the HRDB to those recorded in the EMR for each infusion. Additionally, we selected 14 daily-used PICU drugs and their respective standardized concentration (sedative, analgesic and vasoactive drugs) and compared the correlation between the HRDB and the EMR within the study period (from August 31, 2017, to August 1, 2018).

All analyses were performed after the exclusion of the paired measurements when one of the experimental or reference data was missing. Thus, we intended to differentiate inaccurate data from missing data. A pvalue $<0.05$ was considered statistically significant. Statistical analyses were performed using open access R software (version 3.5.1, 2018-07-02, http://cran.r-project.org/).

Ethics: The study was approved by the institutional review board of Sainte-Justine Hospital (reference number 2016-1210, 4061). The exploitation of the HRDB is regulated by a DB policy validated by the institutional review board and no protected health information were stored in the HRDB nor in the video recordings. No patients or caregivers were recorded in the videos.

\section{RESULT}

Between June 1, 2017, and August 30, 2018, 1378 patients were admitted to the PICU and 100\% were included in the HRDB. During the effective 47 days of study, 81 patients were hospitalized in PICU and 81 $(100 \%)$ were included in the HRDB. Data from 70 patients (86 \%), 295 patients' days, were recorded from medical devices (Table 1) and 4645 data points were video recorded and compared to the corresponding data collected in the HRDB (Table 2).

\section{Monitor data validity}

Statistical analysis showed an overall excellent correlation, agreement and reliability, as shown in Table 2. ICCs were considered as excellent for all the tested variables (Table 2). Bland-Altman analysis showed an excellent accuracy and precision between recorded and collected data within clinically significant pre-defined limits of agreement (Supplemental Digital Content 1). A single heart rate measurement in the experimental data $(0.03 \%)$ was considered as clinically different from the reference data (Figure 2,3). We documented 74 data points $(2 \%)$ that were missing, as detailed in Table 2.

Ventilators' data validity

Statistical analysis showed an excellent overall correlation, agreement and reliability (Table 2, Supplemental 
Digital Content 2). A small, but statistically significant difference was found for the positive inspiratory pressure (mean difference of $-0.022 \mathrm{cmH}_{2} \mathrm{O}$, p-value 0.02 ). This difference was observed only for a minority of the data (95.5\% of all values were equal). Agreement remained over $90 \%$ with an excellent correlation between reference and experimental data. ICCs were considered as excellent for all the tested variables (Table 2). Bland-Altman analysis showed excellent accuracy and precision (Supplemental Digital Content 2). No data were missing (table 2).

\section{Infusion pumps data validity}

The comparison with the data displayed on the infusion pumps showed an overall excellent correlation, agreement and reliability (Table 2) with Bland-Altman analysis showing an excellent accuracy and precision between recorded and collected data for all the tested variables (Supplemental Digital Content 2). ICCs were considered as excellent for all the tested variables (Table 2). Twenty-three infusions (9\%) were not retrieved in the HRDB (Table 2). Nine episodes were related to six patients without any pharmacological data collected in the HRDB and 14 episodes were related to pump dysfunction. Other minor discrepancies were noticed between HRDB and EMR (Table 3). Correlation between HRDB and EMR regarding drugs of interests over the study period were depicted in figure 4 .

\section{Timestamps}

A delay was observed between time synchronized videotapes and collected data from the monitors and the ventilators. This delay was less than 28 seconds and remained stable among patients. Besides, regarding infusion pumps data, we discovered that the data were not collected in the HRDB every 30 seconds as expected, but at different time interval between 10 and 40 seconds or when a modification was done. No delays were observed between the source and the HRDB.

\section{DISCUSSION}

Whether in day-to-day clinical care decision-making or in medical research, the need to evaluate data quality is essential to ensure the reliability of $\mathrm{DB}(9,21,28,29)$. To our knowledge, this is the first study to validate PICU data contained in a specific HRDB $(20,30)$. This article is indissociable from our two previously reports $(8,11)$. The first report described the gathering process of our HRDB $(8)$ and the second gave a comprehensive description of the HRDB's architecture and process (11), this articles constitute the quality assurance of the HRDB $(14,31)$. This third article completes this set. It contributes to the quality assurance phase and to the quality control phase of the HRDB $(14,31)$.

As there were no guidelines specifically designed to guarantee high-resolution data quality $(9,14)$, we elaborated the first complete validation procedure. Our validation procedure was inspired by previously published experiences $(9,10,30,32-34)$ and guidelines $(13-15,28,35)$ regarding data quality assessment in the field of medical DB collected at a lower rate or in a restricted area. To evaluate the quality of the data, we chose to perform an external validation procedure. We compared our extracted results with the information displayed on the monitor or the biomedical device (21). Our study showed an excellent overall accuracy, completeness and reliability of our HRDB when compared to displayed data at the bedside at the same time.

Regarding the accuracy of the dataset, we noticed only one clinically significant different heart rate value. This error was due to a rapid acceleration of the heart rate (Figure 2). In the video, the heart rate increase from 118 beats/minute to 154 beats/minute and the HRDB recorded one single value at 135 beats/minute during the transition. This suggests that monitors processed those data and only refreshed the display at a specific interval (probably between one and two seconds) and did not show intermediate data. Then, the HRDB recorded an intermediate value, which explains the importance of the difference between the reference value and the experimental value. Differences between the HRDB's data and the reference data were observed regarding PIP. Even statistically significant, disagreements were not clinically significant (the maximal difference was $0.5 \mathrm{cmH}_{2} 0$ and concerned only $4.5 \%$ of all the collected PIP, the remaining $95.5 \%$ values were strictly equal) as shown by a mean difference of $-0.022 \mathrm{cmH}_{2} \mathrm{O}$. Only integers are displayed on the ventilator screen and the data processing algorithm of the raw values measured by the ventilator is 
unpublished. Thus, we suspect that these very minor differences may be due to rounding process.

Regarding the completeness of the dataset, $2 \%$ of the data were missing. Even less than previously reported $(9,14,30)$, this number of missing data didn't meet our expectations for this HRDB, as we planned for a $0 \%$ missing data. This loss of data was mainly caused by a systematic error in the data processing. Indeed, we discovered that the original HRDB structure could only record nine parameters simultaneously. Then, when more than nine parameters were sent, the additional data were not registered. Once this issue was identified, we modified our database for an entity-attribute-value structure where each data point is stored as an independent row $(36,37)$.

Regarding infusion pumps and pharmacological data, the discrepancies between the experimental and the reference data or the EMR appeared associated with variability in care more than with a gathering process failure. Regarding the 23-missing data from infusion pumps, we proved that the corresponding infusion pumps were disconnected from the network, thus the data were not sent to the HRDB. This disconnection of the infusion pumps explained these discrepancies between the EMR and the experimental data, with all the pharmacological data missing in six patients. In addition, the large majority of inconsistencies between the EMR and the experimental data were due to a time difference from the beginning or the end of the drug. In the EMR, a drug needs to be ordered before the drug rate could be registered, while in the HRDB, the rate starts to be registered directly when the pump is connected to the network. Furthermore, medications were not registered in the patient EMR, probably because the physician did not order it. However, nursing notes confirmed that the drug was given. In these situations, the HRDB could be considered as more accurate than the EMR. On two occasions, the name of the fluid was different between EMR and HRDB. However, the name recorded on the pump and the one in the HRDB was the same, suggesting the infusion pump drug name was not modified when the medication was replaced. Finally, it happened twice that no data were recorded over a period when they should be. These intervals happened just before the patient was moved to another room and the procedure is to disconnect the pumps before moving the patient. Although these four situations altered the HRDB accuracy, they were not due to a HRDB limitation. Last, timestamp asynchronies were due to a server setting that was corrected after this study.

This study's main limit lies in the lack of validation of the complete dataset $(10,14,30)$. We have considered several procedures to apply either during or after the gathering of the HRDB. Given the gigantic data gathering rate (about 10,000 data points per minute), it is humanly impossible to both gather and validate the data simultaneously while collecting the $\mathrm{DB}$ or even validate the entire database retrospectively. Thus, we decided to perform a point-by-point data analysis on a randomly chosen patients sample considered as representative of the HRDB (30). Besides, some could argue, and they would be right, that we were not able to correct abnormal values or undisplayed data. But, as this dataset is supposed to reproduce the patient's entire course in PICU, abnormal values and undisplayed data should be considered as part of the patient's course as much as a true value (19). Furthermore, this is a study in one institution with an excellent understanding of the value of data quality. Even if the methodology is transferable to other data, this study only validates this particular data in this particular HRDB and its results shouldn't be generalized to other clinically collected data. Finally, even limited as most of the analyzed data were electronically captured, we must consider the possibility of a Hawthorne effect. The observational methodology might have modified the quality of the data being entered in the EMR by the bedside personnel.

\section{CONCLUSION:}

This study showed an excellent overall quality of the data include in the HRDB of our PICU while performing validation procedures on a representative sample. We considered that this study provides an assurance for future HRDB users of the data quality, especially regarding monitor and respirator data. By reporting and detailing this data quality validation process, the process becomes reproducible by any research team and sets a reference for future validation studies of similar datasets.

\section{REFERENCE:}

1. Burykin A, Peck T, Krejci V, et al. Toward optimal display of physiologic status in critical care: I. 
Recreating bedside displays from archived physiologic data. J Crit Care. 2011;26(1):105.e1-105.e9..

2. Mathews SC. The Need for Systems Integration in Health Care. JAMA.2011;305(9):934.

3. Celi LA, Marshall JD, Lai Y, et al. Disrupting Electronic Health Records Systems: The Next Generation. JMIR Med Inform. 2015;3(4).

4. Celi LA, Mark RG, Stone DJ, et al. « Big data » in the intensive care unit. Closing the data loop. Am J Respir Crit Care Med. 2013;187(11):1157-60.

5. Celi LA, Csete M, Stone D. Optimal data systems: the future of clinical predictions and decision support. Curr Opin Crit Care. 2014;1.

7. Brossier D, Sauthier M, Alacoque X, et al. Perpetual and Virtual Patients for Cardiorespiratory Physiological Studies. J Pediatr Intensive Care. 2016;5(03):122-8.

8. Brossier D, El Taani R, Sauthier M, et al. Creating a High-Frequency Electronic Database in the PICU: The Perpetual Patient. Pediatr Crit Care Med. 2018;19(4):e189-98.

9. Johnson S, Speedie S, Simon G, et al. Application of an Ontology for Characterizing Data Quality for a Secondary Use of EHR Data. Appl Clin Inform. 2016;07(01):69-88.

10. Barnes J, Chambers I, Piper I, et al. Accurate data collection for head injury monitoring studies: a data validation methodology. Acta Neurochir Suppl. 2005;95:39-41.

11. Brossier D, Sauthier M, Mathieu A, et al. Qualitative subjective assessment of a high-resolution database in a paediatric intensive care unit-Elaborating the perpetual patient's ID card. J Eval Clin Pract. 2019; In Press.

12. Kahn MG, Brown JS, Chun AT, et al. Transparent Reporting of Data Quality in Distributed Data Networks. EGEMs Gener Evid Methods Improve Patient Outcomes. 2015;3(1):7.

13. Callahan T, Barnard J, Helmkamp L, et al. Reporting Data Quality Assessment Results: Identifying Individual and Organizational Barriers and Solutions. EGEMS Wash DC. 2017;5(1):16.

14. Arts DGT. Defining and Improving Data Quality in Medical Registries: A Literature Review, Case Study, and Generic Framework. J Am Med Inform Assoc. 2002;9(6):600-11.

15. Black N, Payne M. Directory of clinical databases: improving and promoting their use. Qual Saf Health Care. 2003;12(5):348-52.

16. Hall GC, Sauer B, Bourke A, et al. Guidelines for good database selection and use in pharmacoepidemiology research. Pharmacoepidemiol Drug Saf. 2012;21(1):1-10.

17. Black N, Barker M, Payne M. Cross sectional survey of multicentre clinical databases in the United Kingdom. Bmj. 2004;328(7454):1478.

18. Saeed M, Villarroel M, Reisner AT, Clifford G, Lehman L-W, Moody G, et al. Multiparameter Intelligent Monitoring in Intensive Care II: A public-access intensive care unit database. Crit Care Med. mai 2011;39(5):952-60.

19. Johnson AE, Pollard TJ, Shen L, et al. MIMIC-III, a freely accessible critical care database. Sci Data. 2016;3:160035.

20. Goldstein B, McNames J, McDonald BA, et al. Physiologic data acquisition system and database for the study of disease dynamics in the intensive care unit*: Crit Care Med. 2003;31(2):433-41.

21. Feder SL. Data Quality in Electronic Health Records Research: Quality Domains and Assessment Methods. West J Nurs Res. 2018;40(5):753-66. 
22. Revelle W. psych: Procedures for Psychological, Psychometric, and Personality Research. 2018. Available on https://CRAN.R-project.org/package=psych.

23. Sauthier M, Brossier D. BlandAltman: Bland-Altman Analysis and Plot. 2018. Available on: http://github.com/sauthiem/BlandAltman.

24. Bland JM, Altman DG. Statistical methods for assessing agreement between two methods of clinical measurement. Lancet Lond Engl. 1986;1(8476):307-10.

25. Giavarina D. Understanding Bland Altman analysis. Biochem Medica. 2015;25(2):141-51.

26. Gamer M, Lemon J, Singh IFP. irr: Various Coefficients of Interrater Reliability and Agreement. 2019. Available on: https://CRAN.R-project.org/package=irr.

27. Koo TK, Li MY. A Guideline of Selecting and Reporting Intraclass Correlation Coefficients for Reliability Research. J Chiropr Med. 2016;15(2):155-63.

28. Kahn MG, Callahan TJ, Barnard J, et al. A Harmonized Data Quality Assessment Terminology and Framework for the Secondary Use of Electronic Health Record Data. EGEMs Gener Evid Methods Improve Patient Outcomes. 2016;4(1):18.

29. Sukumar SR, Natarajan R, Ferrell RK. Quality of Big Data in health care. Int J Health Care Qual Assur. 2015;28(6):621-34.

30. Shaw M, Piper I, Chambers I, et al. The brain monitoring with Information Technology (BrainIT) collaborative network: data validation results. Acta Neurochir Suppl. 2008;102:217-21.

31. Whitney CW, Lind BK, Wahl PW. Quality assurance and quality control in longitudinal studies. Epidemiol Rev. 1998;20(1):71-80.

32. Adelson PD, Pineda J, Bell MJ, et al. Common data elements for pediatric traumatic brain injury: recommendations from the working group on demographics and clinical assessment. J Neurotrauma. 2012;29(4):639-53.

33. Stow PJ, Hart GK, Higlett T, et al. Development and implementation of a high-quality clinical database: the Australian and New Zealand Intensive Care Society Adult Patient Database. J Crit Care. 2006;21(2):13341.

34. Recher M, Bertrac C, Guillot C, et al. Enhance quality care performance: Determination of the variables for establishing a common database in French paediatric critical care units. J Eval Clin Pract. 2018;24(4):767-71.

35. Siebig S, Kuhls S, Imhoff M, et al. Collection of annotated data in a clinical validation study for alarm algorithms in intensive care - a methodologic framework. J Crit Care. 2010;25(1):128-35.

36. Nadkarni PM, Brandt C. Data extraction and ad hoc query of an entity-attribute-value database. JAMIA. 1998;5(6):511-27.

37. Murphy SN, Weber G, Mendis M, et al. Serving the enterprise and beyond with informatics for integrating biology and the bedside. JAMIA. 2010;17(2):124-30.

Tables:

Table 1: Patients' characteristics

Others: Metabolic, electrolyte disturbance, hematologic, non-cerebral tumor, liver and digestive causes.

Table 2: Comparison between reference data and database data

*All $p$-values were $<0.001$

$* *$ All p-values were $=0$ 
CI95\%: 95\% confident interval

Table 3: Descriptive summary of discrepancies between the database and the electronic medical record

DB: Database; EMR: Electronic Medical Record

Figures:

Figure 1: Data validation process

* Icons made by monkik from www.flaticon.com, ${ }^{* *}$ Icons made by Freepik from www.flaticon.com, *** Icons made by Chris Veigt from www.flaticon.com, **** Icons made by Smashicons from www.flaticon.com.

Figure 2: Correlation and Bland-Altman plot

1. Heart rate. Average bias of $-0.02 \mathrm{bpm}(95 \%$ Confident Interval $+/-0.03)$ and limits of agreement (Average bias +/- 1.96 standard deviation) -1.05;1.01 (95\% Confident Interval +/- 0.05). Acceptable limits of agreement $-5 ;+5 \mathrm{bpm}$.

2. Positive inspiratory pressure. Average bias of $-0.02 \mathrm{cmH} 20$ (95\% Confident Interval $+/-0.02)$ and limits of agreement (Average bias +/- 1.96 standard deviation) -0.23;0.18 (95\% Confident Interval +/0.03). Acceptable limits of agreement $-1 ;+1 \mathrm{cmH} 2 \mathrm{O}$.

Figure 3: Schematic representation of a heart rate curve with a significant acceleration

HR: Heart Rate

Figure 4: Correlation between EMR and HRDB

EMR: Electronic Medical Record, HRDB: High Resolution Electronic DataBase

Supplemental Digital Content:

Supplemental Digital Content 1: Correlation and Bland and Altman analysis for monitors' data.

a. Pulse oximetry. b. Respiratory rate. c. End tidal CO2. d. Systolic arterial pressure. e. Mean arterial pressure. f. Diastolic arterial pressure

Acceptable limits of agreement (+-5\% of the mean of the reference)

Supplemental Digital Content 2 Correlation and Bland and Altman analysis for ventilators' data and for infusion pumps' data.

a. Positive end expiratory pressure. b. Expiratory volume. c. Respiratory rate. d. Minute Ventilation. e. Rate of infusion

\section{Hosted file}

table 1.docx available at https://authorea.com/users/303349/articles/433461-validationprocess-of-a-high-resolution-database-in-a-pediatric-intensive-care-unit-describing-theperpetual-patient-s-validation

\section{Hosted file}

table 2.docx available at https://authorea.com/users/303349/articles/433461-validationprocess-of-a-high-resolution-database-in-a-pediatric-intensive-care-unit-describing-theperpetual-patient-s-validation

\section{Hosted file}


table 3.docx available at https://authorea.com/users/303349/articles/433461-validationprocess-of-a-high-resolution-database-in-a-pediatric-intensive-care-unit-describing-theperpetual-patient-s-validation
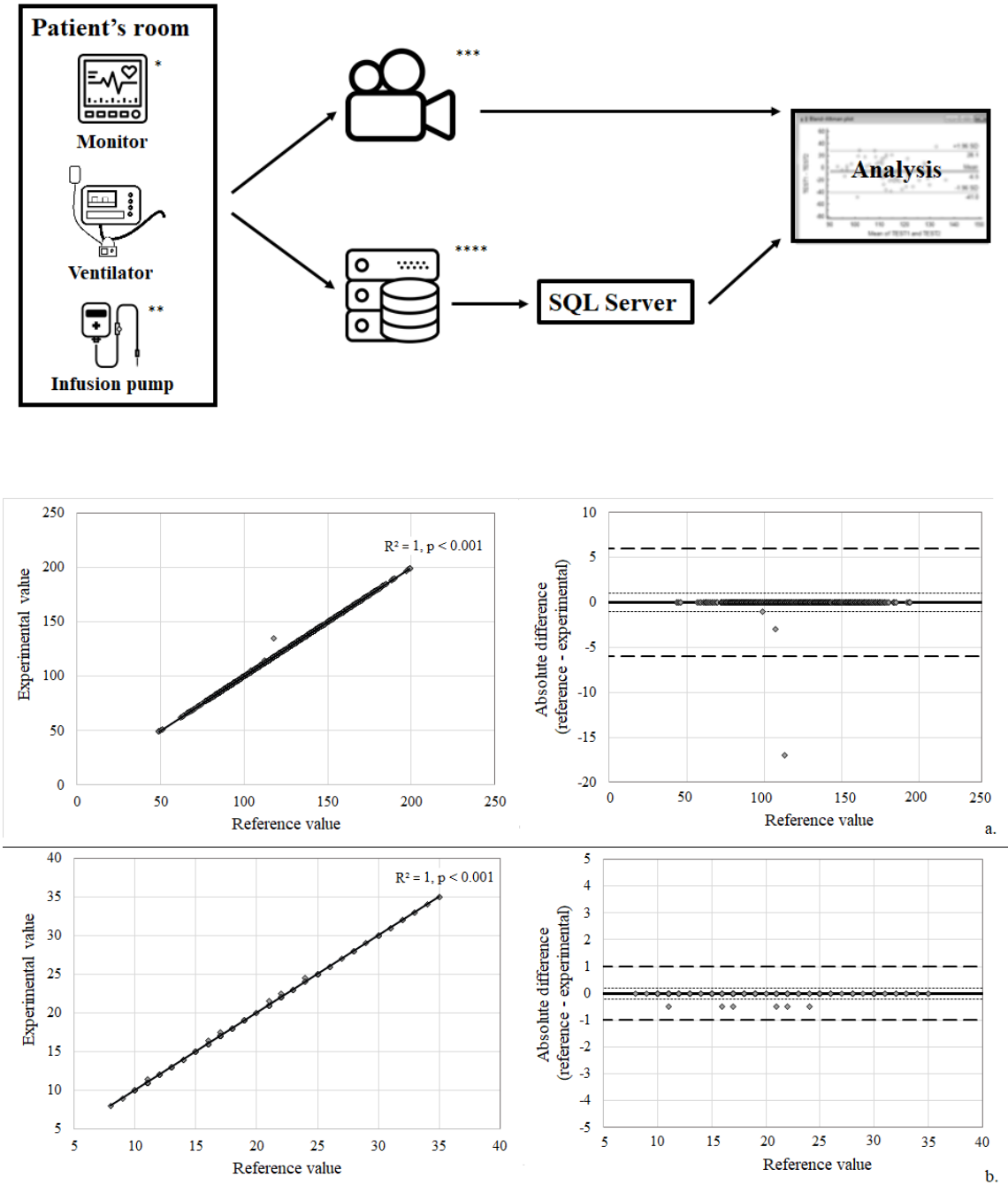

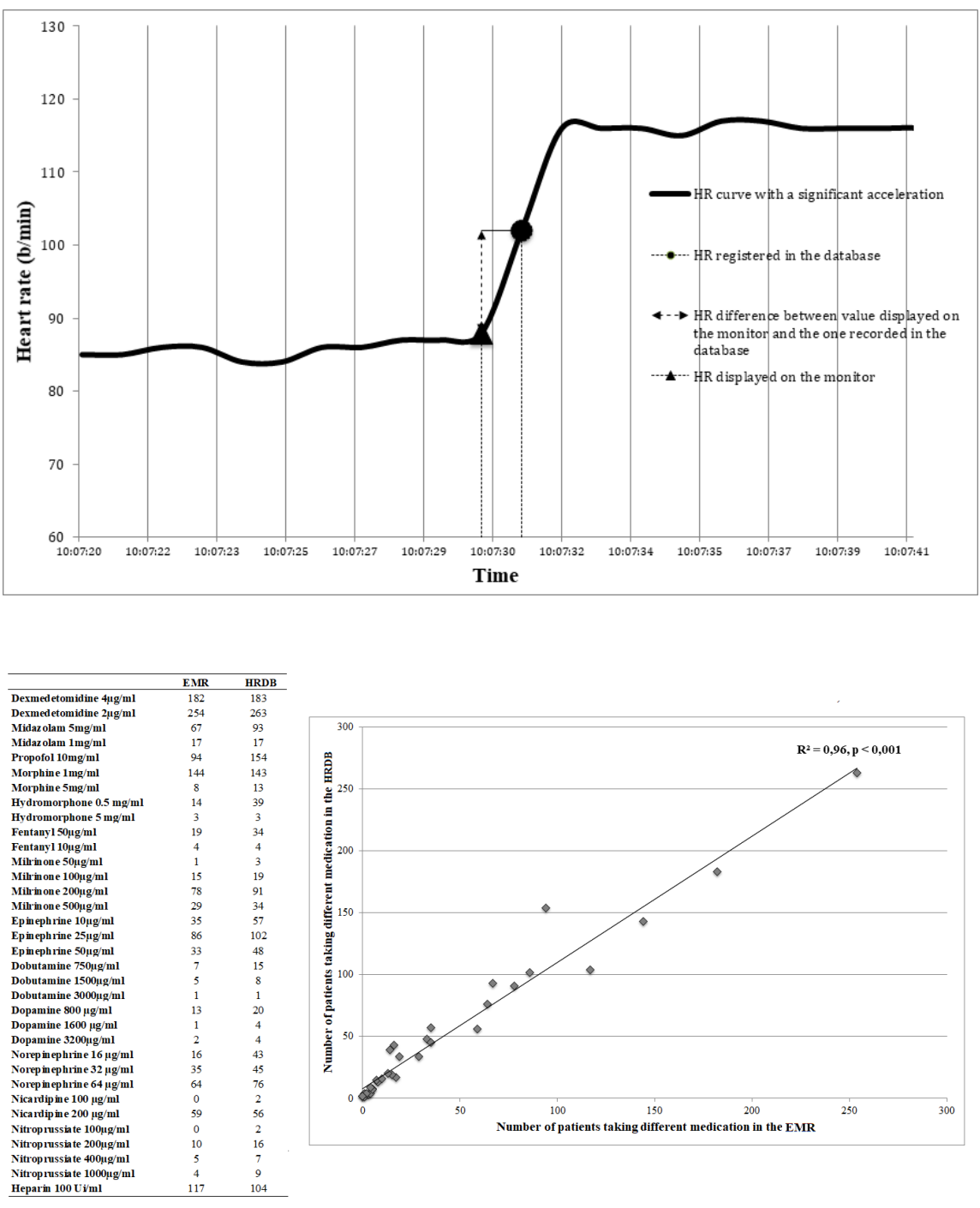